\title{
New Trends in Astronomy Teaching
}

\author{
By L.E. Abati \\ Società Astronomica Italiana, Unità di Ricerca CNR “Asiago", c/o Department of Astronomy \\ Vicolo Osservatorio, 5 - 35122 Padua, Italy
}

We are all aware of the fact that Astronomy teaching is not an easy task for many different reasons which we are going to examine during this Colloquium. The present contribution focuses on one of these reasons we consider of major importance for Astronomy in the school: Teacher Training.

Teacher training has been debated extensively for a long time and discussion is being presently livened up.

Institutions and associations are promoting research, studies and comparisons on this issue. For instance, the Osnabrück conference "Teacher Education in Europe: Evaluation and Perspectives" (June 1995) - the International Forum of Rome (September 1995) and, specially devoted to Astronomy, the EU/ESO Workshop "Astronomy teaching in the European secondary school "(Garching, 1994), SAIt Workshop in Reggio Calabria "European Science Teacher Training "(September 1995), Conferences of Teaching Astronomy in Spain, the Constitutional Conference of the European Association for Astronomy Education (EAAE, Athens, 1995).

It is difficult to treat Astronomy teacher training without including it in a more general context. Teacher training does not only mean providing teachers with suitable teaching skills for each subject. First of all, teachers should bear in mind the interaction with a social and cultural reality that may affect learning processes. And the educational (and teaching) system is not neutral to the external framework. European and non-European countries have their own national differences with different school systems and choices made in the field of teacher training. Time does not allow us to go in detail into a comparison of the various solutions adopted in different countries. However there are some common elements in teacher training both in terms of organization and content (Osnabrück Conference, ESO Workshop, EURIDICE '90, EURINS), see Fig. 1.

\section{Common organizational elements $\dagger$}

- Training often in post-secondary institutes, not necessarily in universities

- Possible training both in specialized institutes and universities

- Curricula of specialized institutes mainly with an educational background, lead to teaching in compulsory schools

- University curricula mainly with a specialized background in one or two disciplines, often leads to teaching in secondary schools (Dutch model)

- No specific curricula leading to teaching in Universities

- In-service training and requalification linked to initial training often in different centres

- Refresher courses compulsory almost everywhere

- Common changes of the institutional mechanism referring to teacher training

\section{Figure 1.}

$\dagger$ with the exception of Italy 
COMMON ELEMENTS

\begin{tabular}{|c|c|}
\hline UNIVERSITY & 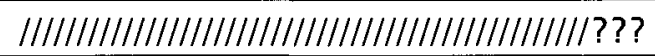 \\
\hline SECONDARY SCHOOL (High School) & 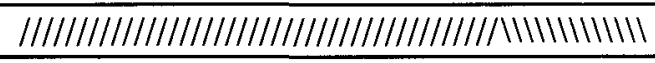 \\
\hline SECONDARY SCHOOL (Junior H. S.) & 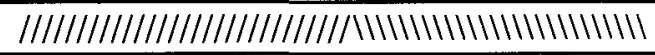 \\
\hline PRIMARY & 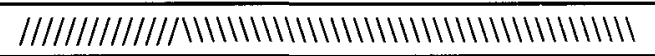 \\
\hline NURSERY & 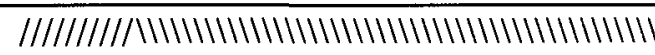 \\
\hline
\end{tabular}

\section{IIIIIIIIIII subject knowledge \\ IIIIIIIIII educational skills}

Figure 2. Taken from the presentation of G. Luzzato, President of the Italian Conference of the Interdepartimental Centres for Educational Research (CONCIRD) given at the GIREP '95 held in Udine, Italy, August 1995.

Concerning content, Fig. 2 is extracted by considering teachers' degree of specialization and teachers training curricula.

It shows the relative proportion of subject knowledge and educational skills at various school levels. Almost everywhere, outside this conference, it is still believed that at university level the qualification for teaching is automatically guaranteed by a competence in research.

We all agree that good astronomy teachers should be competent both in subject knowledge and educational skills. Therefore, the professional profile of teachers should combine the two aspects. This implies that, in teacher training, disciplinary courses and educational courses should converge in subject didactics which then interface with school practices as shown in Fig. 3, also taken from the above mentioned work.

Astronomical didactics becomes the valuable bridge between the disciplinary themes and the educational ones. In the subject didactics, competences deriving from different areas should converge.

Features emerging from an analysis of the teacher training system show a general dissatisfaction concerning the actual integration between subject and educational science which are located in separate departments often scarcely communicating with each other. 
UNIVERSITY

\section{SUBJECT DEPARTMENT EDUCATION DEPARTMENT}

Disciplinary
courses
Educational

courses

Subject

didactics

\section{SCHOOL SYSTEM}

School practice

\section{Figure 3. Suggested Devision of Teacher Education}

For teaching purposes, one must consider that scientific knowledge is increasing and changing very rapidly, too. Most of what we are going to hear at the next IAU Assembly in the field of Astronomy could hardly be imagined at the time when a person, today in his forties/fifties, was attending University. The same can be applied to students. Therefore, the need is to focus on skills and on the development of abilities, on flexibility and basic training, as also stressed by George Greenstein, and astronomy seems to be particularly suitable for this purpose. Moreover, efficient refresher courses play a key-role in teaching. It should be noted that the institutional training and requalification system is subject to change almost everywhere. But the discussion would shift towards matters of educational policy, scientific, cultural and administrative independence of schools. This is not the most suitable place to tackle such issues. Our aim is to consider the role that Astronomy can take on in an educational system.

\section{SOCIAL AND POLITICAL ELEMENTS}

- Cultural

Scientific Independence of schools

Administrative

- Legal status of teachers

- Careers depending on the quality of service

- Flexible curricula curricula updating curricula adjusting to different situations

\section{Figure 4. Social and Political Elements}

Astronomy is a discipline rooted in the early culture of "man-anthropos". Therefore, tracing its history means tracing the main stages of the development of human thought and knowledge. Since the most ancient evidence of man's interest in the universe, such as primitive graffiti as well as theoretical approaches of the oldest schools of thought (Chaldean, Egyptian, the Pythagorean School and pre-Columbian civilizations), it is possible to find a constant reference to two paradigms: 1) a rational knowledge based on the "scientific" observation of the phenomenon; 2) a "magical", "mythological" aspect involving the emotional, irrational sphere, the individual and collective imaginary world. In front of the starry sky, man feels "sub divo", immersed in mystery, in the arcane. The first paradigm has lead to the technical applications of astronomical knowledge, first of all in time measurement based on the cyclical trend of physical and biological phenomena (from tides to recent biorhythms). Historically, these applications precede the theoretical elaboration and systematization which is continued in modern astronomical research up to its most complex fomulations. Just think of cosmology, of the theory of evolution of 
Universe, until the recent discoveries in the solar system. After all, in the sciences, practical aims always come before theory (a typical example is geometry which was invented as a response to the need for measuring distances on the ground). The second paradigm led to all the intuitions, interpretations, experiences which converged in the pre-Galilean culture of the so-called pseudosciences, among which astrology (compared to astronomy) represents the attempt to establish a direct connection between the cosmic phenomenon and the individual and social existence. The reference to these two paradigms allows us to place astronomy in a privileged position in a modern scientific context. Until Positivism, in fact, what seemed to be an insurmountable distinction between "science" and "myth" existed and was based on Cartesian and Galilean assumptions: scientific knowledge excluded, by definition, the whole mythological heritage.

However, more recently, after questioning some "scientific" certainties (in mathematics and physics) and revaluing what is "indeterminate" and "probable" with respect to the strict "cause and effect" relationship (determinism, probabilism, the laws of chaos), scientific and mythological approaches no longer clash. Nowadays, they are considered two separate but integrated ways of experiencing and exploring reality. This vision is also supported by a biological approach, as more recent studies in neuroscience have proved: the two cerebral hemispheres are entrusted with different but integrated functions and are not subordinated one to another, according to the obsolete principle of "cerebral dominance". For example, in listening to music, the perception of notes and their sequence depends upon the function of the left hemisphere, but our perception of melody and impression is due to the right hemisphere.

In the creation of scientific knowledge and in that "breeding ground" called school, of course, both ways of knowledge are always present. The teaching criteria, in fact, should represent and implement the two approaches.

At this stage, we can say that astronomy is included with a special educational value in school curricula, since it is one of the few, if not the only, subject that maintains a double link:

a) with the most advanced research modalities complying with modern epistemological criteria, according to the well known Popperian statements, which have enabled achievement of present technological results and applications;

b) with an attractive and "magical" background that has an immediate hold over irrational but all the more important aspects in the acquisition of knowledge. Let us think of science fiction, which does not mean a "fantastic science", but a science combined with fantasy, where astronomy has a leading position; think of the appeal of equipment, telescopes, radiotelescopes, space probes, satellites, etc.

From a strictly educational point of view, we must bear in mind that teaching is above all communication and, as such, must keep in touch with the different aspects of personality, with the cognitive-rational sphere and the emotional one. Modern neuroscience is developing identification of these aspects in their biological substratum (cortex and sub-cortical structures - limbic lobe). School curricula should follow and take into account this basic ability to communicate and interact. In this interaction, there is space for all theories of communication (rational sphere) and relational dynamics studied by psychological sciences (emotional sphere).

Astronomy is suitable for such modular knowledge and its introduction in all school curricula should promote the most complete education of the individual. Therefore, we are allowed, on a scientific basis, to say that the value of astronomy is twofold:

- Informative: indispensable knowledge in a modern culture which is concerned (for biological reasons, too) about protecting the planet;

- Formative: it is a subject that involves different levels of individual and social knowl- 
edge, thus representing a model of integration between cognitive-rational modalities and structures and emotional-irrational ones. Recognizing these two values and clarifying the function of astronomy in school, that is interacting with students at an emotional level too, and using the appeal of a starry sky to guide them towards a scientific understanding of the world, can be considered a very successful new trend in astronomy teaching, that no other school subject can achieve.

Experience from cooperation in school programme projects has pointed out that a teaching programme is justified if, besides the content, the aims, the learning objectives and educational methodology are made clear. Of course it is always important to take into account the natural ideas of the students.

We believe that the aim of teaching astronomy in all schools is to understand that we are on the Earth but we live in the Universe. As generally astronomy is not an independent subject at school, there should be astronomy "modules" during teacher training in didactics of natural science and physics, the aims of which could be:

- the consciousness of Earth as a complex system in dynamic balance within the solar system (science) as well as

- understanding the universality of the physical laws from human level to microcosm and macrocosm (physics) with the objective of

- locating the Earth in space and time and setting the fundamental steps of its evolution.

As for methodological indications we think that, at all school levels, we do not have to teach the history of astronomy, but with the cognitive instruments of our students, try to explain the world around us. However, the formal introduction of astronomy in school curricula is not sufficient to guarantee the teaching of astronomy and particularly a correct teaching of it. The greatest effort in teaching astronomy has to shift attention from the curricula to the training of teachers. It is also a common experience that young people are interested in the phenomena surrounding them, rather than in their academic explanation. Students read newspapers, watch TV, sit at workstations watching the WWW, visit science museums, planetaria. In other words, we have to consider that school is not the only educative agency nowadays.

In Italy a lot of activities concerning astronomy teaching are organized by many observatories and astronomy departments and by the Italian Astronomical Society, of which I would like to give you some examples. For a long time the Italian Astronomical Society (SAIt), a free association of professional astronomers, amateurs and teachers, has set up an Educational Committee consisting of university professors and teachers. This commission is a sort of concentration nucleus for the activities concerning astronomy teaching; it regularly interacts with the public authority, the other scientific societies, the teachers associations; it keeps astronomy to the fore in the science curricula; it works out curriculum proposals, it offers modular courses for teachers. It arranges summer courses and promotes competitions in schools, and text-books analysis; and it brings out educational material.

Problems in teaching astronomy: there are many problems concerning astronomy teacher training which we are not going to treat here exhaustively, but just mention a few of them common to other disciplines: initial teacher training, in-service teacher training, curricula, etc. For Astronomy we have to add some other specific problems:

- Astronomy is present in other university curricula, physics and natural science

- Astronomy teachers need broad competence

- Research in astronomy education is very poor

- Time and Space are peculiar.

The latter could be considered as a drawback, but in my opinion it represents a great 
advantage for astronomy since it could guide students to respect time, space and, more generally, the modality of the natural world.

I hope I have been able to convince you of the fact that, as mentioned at the beginning of this presentation, teacher training does not only mean providing teachers with suitable teaching skills for each subject. Above all, teachers should be aware of the interaction with students in a social and cultural reality which can affect learning processes. The basic objective should aim at recovering an interpersonal relationship that reproposes the fundamental values of true humanism in the scientific universe. Historically, these values have never been set aside. Therefore we believe that if we want astronomy in the school, we have to take care not only of curricula and structures, but, most of all, of teachers, in their initial training, in their in-service training and in practical training. Institutions and parties in general which care for astronomy in the school and with the school have to move in this direction for the future of our society and of man.

\section{REFERENCES}

Luzzato, G. 1995, Proceedings of GIREP '95, Udine.

Teacher Education in Europe, Evaluation and Perspectives, 1995, Proceedings of the Osnabrück Conference.

Workshop on European Science Teacher Training, 1995, in press, SAIt, Reggio Calabria.

Astronomy teaching in European secondary schools, 1994, Proceedings EU/ESO Workshop, Garching.

Scuola di Specializzazione all'Insegnamento secondario, 1994, CIRE, Bologna.

Itinerari astronomici, 1993, Itinerari Educativi, Venice.

ABATI, L. 1995, Il Giornale di Astronomia no.2, SAIt.

Initial Teacher Training, 1991, Eurdid Station Unit. 